\title{
The Validity of Open-Source Elevations for Different Topographic Map Scales and Geomatics Applications
}

\author{
Gomaa M. Dawod1 (), Ibrahim E. Ascoura² \\ ${ }^{1}$ Survey Research Institute, National Water Research Center, Giza, Egypt \\ ${ }^{2}$ Umm Al-Qura University, Makkah, Saudi Arabia \\ Email: dawod_gomaa@yahoo.com, ieascoura@uqu.edu.sa
}

How to cite this paper: Dawod, G.M. and Ascoura, I.E. 2021) The Validity of OpenSource Elevations for Different Topographic Map Scales and Geomatics Applications. Journal of Geographic Information System, 13, 148-165.

https://doi.org/10.4236/jgis.2021.132009

Received: February 20, 2021

Accepted: March 16, 2021

Published: March 19, 2021

Copyright $\odot 2021$ by author(s) and Scientific Research Publishing Inc. This work is licensed under the Creative Commons Attribution International License (CC BY 4.0).

http://creativecommons.org/licenses/by/4.0/

\begin{abstract}
This paper presents an analysis of four open-source Global Digital Elevation Models (GDEMs) and compares them on two topographic profiles (nearly flat, and hills regions) for mapping and geomatics applications. The chief intention is to investigate if GDEMs-based heights, contour intervals, slopes, and topographic profiles are valid for all map scales of topographic mapping, which constitutes a major issue in mapping activities. Two case studies, the Nile delta in Egypt and Makkah city in Saudi Arabia, have been utilized to represent flat and moderate-topography patterns. The investigated GDEMs include the most-recent released models: ASTER v.3, ACE 2, SRTMGL1 v.3, and NASADEM_HGT v.1 released in 2019 and 2020 with spatial resolutions of 1 and 3 arc seconds. Available accurate Ground Control Points (GCP) consist of 540 stations in the Nile delta and 175 stations in Makkah. Based on the available datasets in two study areas, it has been found that the accuracy of investigated GDEMs over known checkpoints ranges from \pm 2.5 and \pm 5.1 meters in the Nile delta region, while it varies between \pm 5.1 and \pm 8.0 meters in the Makkah area. That indicates that the utilization of GDEMs in topographic mapping differs significantly between flat and hilly spatial regions. Therefore, it is recommended to avoid using GDEMs for developing topographic maps of scale 1:25,000 or larger in flat regions and map scale 1:50,000 or larger in hilly regions. Additionally, the accomplished results showed that all GDEM-based slopes do not match with the actual slopes from known GCP over cross section's length up to 30 kilometers. Thus, it is concluded that GDEMs are not the appropriate heights' source for topographic mapping at medium and large map scales, and could not be utilized for topographic profiling in precise engineering and geomatics applications.
\end{abstract}




\section{Keywords}

Topographic Maps, GDEMs, Heights, Map Scales, Contour Interval

\section{Introduction}

Topographic maps represent a chief type of maps utilized in numerous geomatics applications, such as engineering projects, hydrological modelling, disaster risk assessment, geomorphological and hydrogeological analysis, and environmental applications. Contour lines represent a vital piece of information that appeared on printed and digital topographic maps. They represent the terrain topographic variations and enable estimating heights of required random points. Nowadays, the most frequent form of storage and analyzing height datasets is the Digital Elevation Model (DEM) digital format. It is known that heights used to generate contour lines could be obtained from a wide range of sources including terrestrial surveying, Global Navigation Satellite Systems (GNSS) data, aerial photos, satellite images, and GDEMs. On another hand, Geographic Information Systems (GIS) enables handling, processing, and analyzing DEMs to generate many forms of mapping terrain topography including generating contour lines for a wide range of applications. Some of such applications include the production of flood hazard maps [1], mapping of geological structural lineaments [2], analysis of fluvial landscape in mountainous regions [3], monitoring impacts of sea level rise [4], the production of topographic maps [5], and geoid modelling [6].

In many developing countries, precise national DEM models might be existed but unpublished for public usages. So, researchers usually utilize the available open-source free GDEMs. In the last few decades, many GDEM models, with variable coverages and spatial resolutions, have been produced and made freely accessible over websites. Examples of such GDEMs include the Shuttle Radar Topography Mission (SRTM), the Advanced Spaceborne Thermal Emission and Reflection Radiometer (ASTER), EarthEnv-DEM90, the Global Multi-resolution Terrain Elevation Data 2010 (GMTED2010, the Global Land One-km Base Elevation (GLOBE), the Global 30 arc-second (GTOPO30), and the Advanced Land Observing Satellite (ALOS)-based World 3D - 30 m (AW3D30).

The accuracy of open-source GDEMs constitutes a vital role in their utilization for mapping and environmental studies. Thus, many research studies have been focused on estimating the precision and accuracy of GDEMs. For example, Dawod and Al-Ghamdi [7] have examined the reliability of eight GDEM models for geomatics applications in both Egypt and Saudi Arabia. The results showed that their accuracy, in terms of standard deviation over checkpoints, ranges between \pm 1.9 and \pm 6.6 meters. Similar results have been reported in other regions such as the USA [8], India [9], and China [10].

Rather than the printed or digital topographic maps, several geomatics appli- 
cations, particularly utilizing GIS, rely on a digital representation of the topography. Such activities need a definite level of height accuracy depending on its objectives and the spatial extent of the study area. Using GDEMs might not appropriate for high-accuracy geomatics applications. A typical example of such a situation is the impact assessment of Sea Level Rise (SLR) in small or moderate regions. It is known that the average anticipated SRL on a global basis in 2081-2100 is estimated to range between 0.40 and 0.63 meters relative to the 1986-2005 level [11]. Monitoring and estimating risk impacts of such small level rise necessitate a high-accuracy terrain representation. Abdel-Aziz et al. [12] have investigated the reliability of a few GDEMs in delineating inundated regions due to sea level rise over the Nile delta area. It has been found that the reliability of three investigated global models, when compared to a high-accuracy local DEM, is less than 20\%. Similar results have reported by other researchers in other regions [13]. Another example is the utilization of low-accurate GDEMs in engineering applications. Zhang and Chu [14] emphasized that precise quantification of surface depression storage depend significantly on DEM-based topographic characteristics principally the vertical accuracy and spatial resolution. On the other hand, there exist some geomatics applications that could be performed utilizing the few-meters accuracy of GDEMs. Reconnaissance, project planning for medium or large spatial regions, hydrological modelling, surface runoff estimation, and geoid modelling could be examples of such activities.

The current research study aims to investigate the absolute accuracy and the validity of elevation data from different sources for contour generation at several topographic map scales. Additionally, it investigates two other relative topographic factors, namely slope and cross-sections, employed broadly in numerous geomatics applications. This comprises a vital concern in topographic mapping to investigate if GDEMs-based contours and topographic profiles could be utilized for all map scales and geomatics applications. As far as the authors' concern, it might be the first research study, at least in Egypt, to tackle such a significant subject.

\section{Standards and Specifications of Topographic Maps}

Standards and specifications have been developed worldwide for contour lines characteristics including the accuracy of collected data and the methods of representing such important lines. Contour intervals to be shown on a map vary with the required map scale. The term contour interval represents the vertical distance or the elevation difference between two successive contour lines. The scale of a map is the ratio between the length of a line on the map and its actual length on the ground. Traditionally, topographic maps are drawn on medium map scales such as 1:5000, 1:10,000, 1:25,000, and 1:50,000. Generally speaking, selecting the appropriate contour interval for a specific map depends on several factors including the map scale, the extent of the surveyed area, the nature of the topography, and the number and distribution of available height dataset, and the 
purpose of the map itself [15]. A rule of thumb is that the appropriate contour interval on a map might be estimated as three times the Root Mean Square Error (RMSE) of the utilized input height data [16]. The vertical accuracy is represented by RMSE of the utilized height datasets as:

$$
\mathrm{RMSE}=\sqrt{\frac{\sum_{1}^{n}\left(P_{i}-O_{i}^{2}\right)}{n}}
$$

where $P_{i}$ and $O_{i}$ are the predicted and observed height respectively of point $i$, and $\mathrm{n}$ is the total number of points.

Examples of standards and specifications of topographic maps, particularly for contour lines' characteristics, are summarized herein. The Australian map specifications state that the minimum elevation accuracy of datasets to generate contour lines are 5, 10, 25 meters for map scales 1:25,000, 1:100,000 and 1:250,000 respectively [17]. In Burundi, the contour intervals for 1:5,000 and 1:25,000 topographic maps equal 5 and 10 meters respectively and the vertical accuracy of these contour lines are less than 2.5 and 5.0 meters respectively [18]. Additionally, the suggested contour intervals in India for 1:5,000, 1:10,000 1:25,000, and 1:50,000 maps equal 1.25, 5.0, 8.0, and 15 meters respectively [19]. Also, the US Geological Survey (USGS) state that the vertical accuracy standard requires that the elevation of 90 percent of all utilized height points must be correct within half of the contour interval [20]. Additionally, the map specifications of the State of Qatar defined the appropriate contour intervals of $2 \mathrm{~m}, 5 \mathrm{~m}$, and $10 \mathrm{~m}$ for map scales 1:5000, 1:10,000, and 1:200,000 respectively. Additionally, the RMS of heights used in topographic mapping is one-third of the required contour interval [21].

Nationally, the Egyptian Survey Authority (ESA) has published in the year 2020 the updated specifications of topographic maps [22]. For topographic maps' contour lines, the Egyptian standards state that the RMSE of utilized datasets to generate contour lines should be less than one-third of the contour interval. The utilized contour intervals for map scales 1:10,000, 1:25,000, 1:50,000, 1:100,000, and 1:250,000 are 2, 5, 10, 20, 50 meters respectively. If contour lines are not displayed on map scales 1:5,000 and 1:2,500, spot heights should be plotted on every 100 and $50 \mathrm{~m}$ horizontally with vertical accuracy levels equal 0.75 and 0.40 meter respectively. To conclude this issue, Table 1 presents typical examples of contour interval and vertical accuracy used in producing topographic maps with several map scales worldwide.

The relief, slope, and cross-sections constitute important factors in characterizing the topography of a spatial region [23]. The relief, $R$, is the height difference between the maximum $\left(H_{\max }\right)$ and minimum $\left(H_{\min }\right)$ heights of the region. The slop between two points is their height difference divided by the horizontal distance between them. The slope could be evaluated in the units of degrees or as a percentage (Equation (3)):

$$
R=H_{\text {max }}-H_{\text {min }}
$$


Table 1. Typical standards of topographic maps.

\begin{tabular}{ccc}
\hline Map Scale & Contour Interval $(\mathrm{m})$ & Vertical Accuracy of Utilized Datasets $(\mathrm{m})$ \\
\hline $1: 5000$ & 1.25 & \pm 0.4 \\
$1: 10,00$ & $2.0 / 5.0$ & $\pm 0.7 / \pm 1.7$ \\
$1: 25,000$ & $5.0 / 8.0 / 10.0$ & $\pm 1.7 / \pm 2.6 / \pm 3.3$ \\
$1: 50,000$ & 10.0 & \pm 3.3 \\
$1: 100,000$ & $10.0 / 20.0$ & $\pm 3.3 / \pm 6.7$ \\
$1: 250,000$ & $25.0 / 50.0$ & $\pm 8.3 / \pm 16.7$ \\
\hline & $S=\frac{\Delta H}{D} \times 100$ &
\end{tabular}

Additionally, a new factor called Slope Validity $(S V)$ is proposed, herein, to compare the performance of GDEM-based estimated slopes when compared to precise slopes. It is computed as the percentage of the slope, between two points, from both GDEMs and known accurate heights at those checkpoints, as:

$$
S V_{\mathrm{GDEM}}=\frac{S_{\mathrm{GDEM}}}{S_{\text {known }}} \times 100
$$

Concerning the terrain topography, it could be divided into classes according to several factors such as the slope. Table 2 presents an example of slope-based topography categories [24] [25].

\section{Methodology and Available Data}

The utilized GDEMs models, herein, include NASADEM_HGT v. 1, SRTMGL1 v. 3, ASTER v. 3, and ACE2 models. They constitute the most-recent global DEM models at the time of conducting this research. Table 3 summarizes the characteristics of those selected global DEMs, including:

1) SRTM: The Shuttle Radar Topography Mission (SRTM) is a global DEM, that has two versions: SRTM1 with a spatial resolution of 1 arc second, i.e., approximately 30 meters, and SRTM3 model with a 3 arc second resolution. SRTMGL1 v. 3 [26] has been utilized herein. (download from e.g. https://earthexplorer.usgs.gov/).

2) ACE2: The Altimeter Corrected Elevations, Version 2 is a global digital elevation model created by synergistically merging the SRTM data set with Satellite Radar Altimetry within the region bounded by $60^{\circ} \mathrm{N}$ and $60^{\circ} \mathrm{S}$. ACE2 was developed at resolutions of 3, 9 and 30 arc-seconds, and 5 arc-minutes [27]. The 3" ACE2, utilized herein (downloaded from https://sedac.ciesin.columbia.edu/data/set/dedc-ace-v2).

3) ASTER: The Advanced Spaceborne Thermal Emission and Reflection Radiometer (ASTER) is a 1 arc-second global DEM [28]. The ASTER version 3 has been publically released in 2019 (download from e.g.

https://asterweb.jpl.nasa.gov/gdem.asp). 
Table 2. Topography groups based on slope.

\begin{tabular}{ccc}
\hline Topography Class & Slope Type & Slope $\%$ \\
\hline Lowlands & Gentle & $<3 \%$ \\
Plateaus & Moderate & $3 \%-8 \%$ \\
Upper terraces & Moderate & $8 \%-12 \%$ \\
Hills & High & $12 \%-40 \%$ \\
Mountains & Very High & $>40 \%$ \\
\hline
\end{tabular}

Table 3. Characteristics of the utilized global DEMs.

\begin{tabular}{cccc}
\hline \multirow{2}{*}{ DEM } & Released Year & \multicolumn{2}{c}{ Spatial Resolution } \\
\cline { 3 - 4 } & & Arcsecond & Meter \\
\hline ASTER v.3 & 2019 & $1^{\prime \prime}$ & $\sim 30$ \\
ACE2 & 2019 & $3^{\prime \prime}$ & $\sim 90$ \\
SRTMGL1 v. 3 & 2019 & $1^{\prime \prime}$ & $\sim 30$ \\
NASADEM_HGT v. 1 & 2020 & $1^{\prime \prime}$ & $\sim 30$ \\
\hline
\end{tabular}

4) NASADEM_HGT v. 1: A GDEM developed by the US National Aeronautics and Space Administration (NASA) in collaboration with German and Italian agencies, and released in 2020. It is based on improved processing and merging of several data sources such as SRTM, ASTER, Ice, Cloud, and Land Elevation Satellite (ICESat) and other sources [29]. It can be downloaded from https://search.earthdata.nasa.gov/.

The main study area is the Nile delta region, Egypt. It extends from Damietta city on the east to Alexandria city along the Mediterranean sea and extends to the south to Cairo (Figure 1(a)). 540 GCPs with accurate heights, within \pm 0.05 meter, have been collected in the Nile delta. The four GDEMs have been downloaded, from their individual website, for this region. First, the topography pattern of the region has been investigated by utilizing the Arc GIS 10 software to convert GDEM-based heights into slope percentages. It has been found that the average slope of the Nile delta equals $3.1 \%$, (Figure 1(b)) which indicates that its topography could be considered as a moderate or plateaus class (Table 2). Consequently, another study area has been added. It represents Makkah city in the Southwest region of the Kingdom of Saudi Arabia (KSA) about $70 \mathrm{~km}$ east of Jeddah city on the Red sea (Figure 2(a)). 175 GCPs with accurate heights, within \pm 0.05 meter, have been collected in Makkah. Similarly, the four GDEMs have been downloaded and the topography slopes have estimated (Figure 2(b)). It has been found that the average slope of the Makkah equals 17.4\%, which indicates that its topography could be considered as a high or hills class.

Having downloading spatial grids of the four investigated GDEMs for both study areas (Figure 3 and Figure 4), their interpolated heights at the available GCPs have been compared to the known precise heights utilizing the Arc GIS 10 package. The accomplished results are presented and investigated in the next section. 


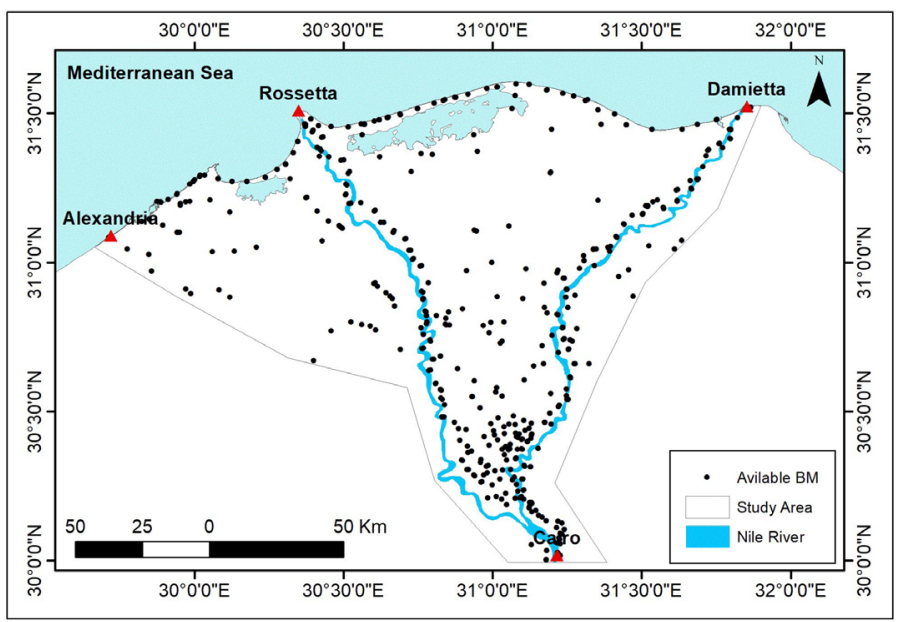

(a)

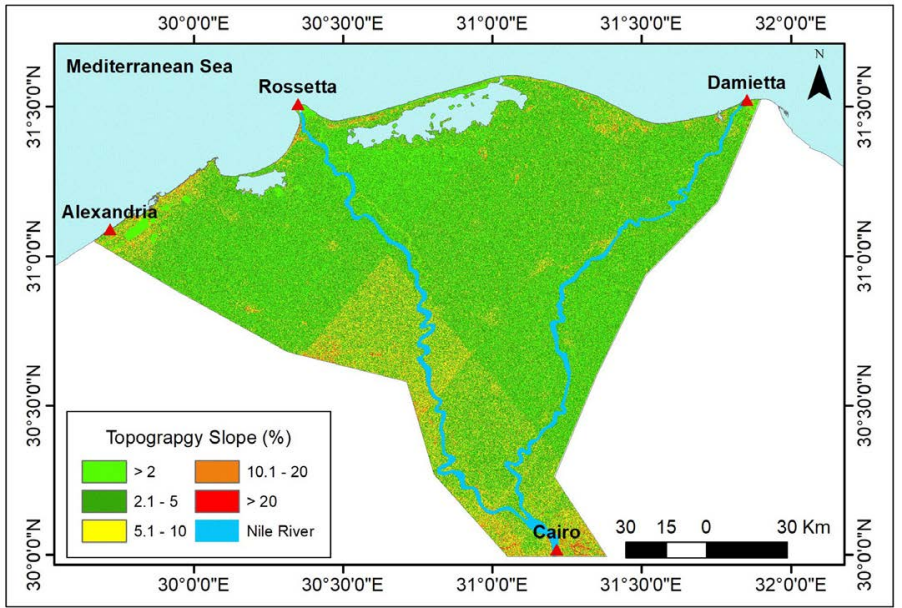

(b)

Figure 1. Nile delta study area. (a) Available data; (b) Topography.

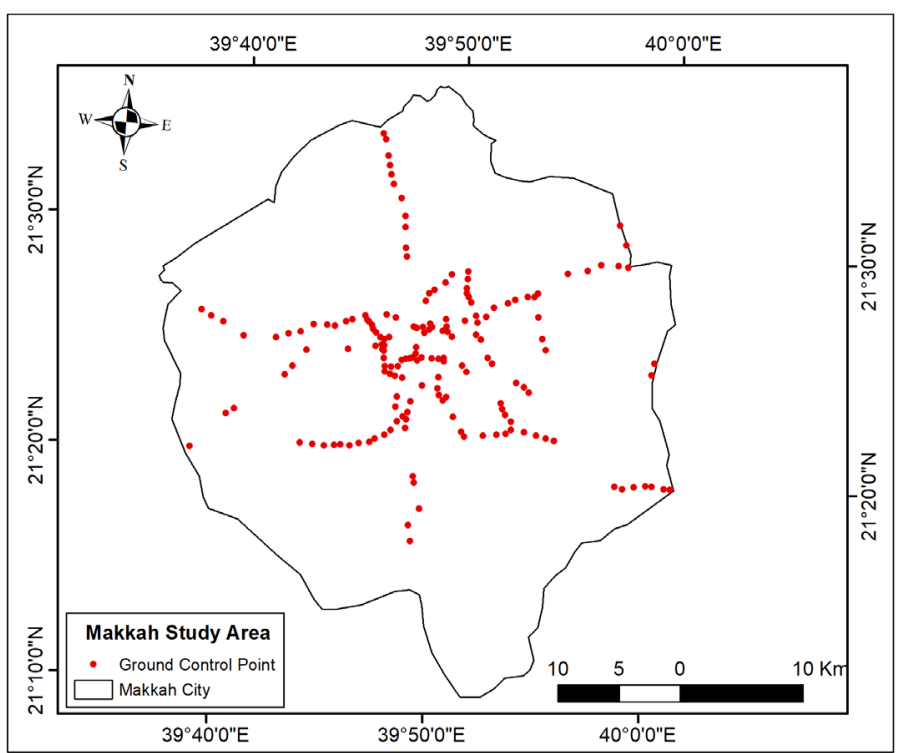

(a) 


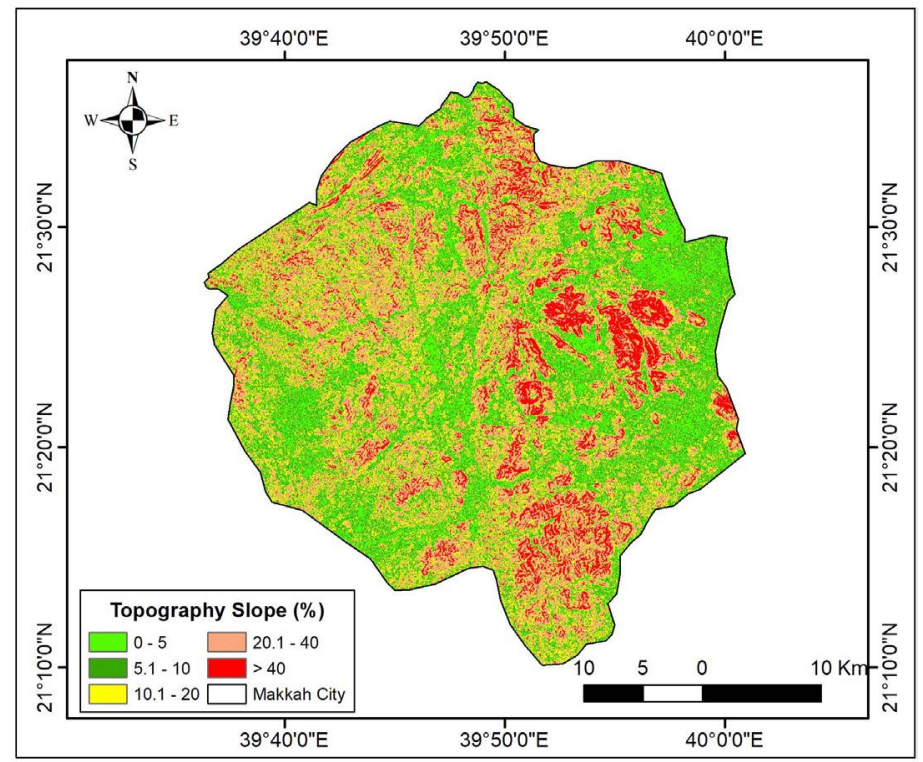

(b)

Figure 2. Makkah study area. (a) Available data; (b) Topography.

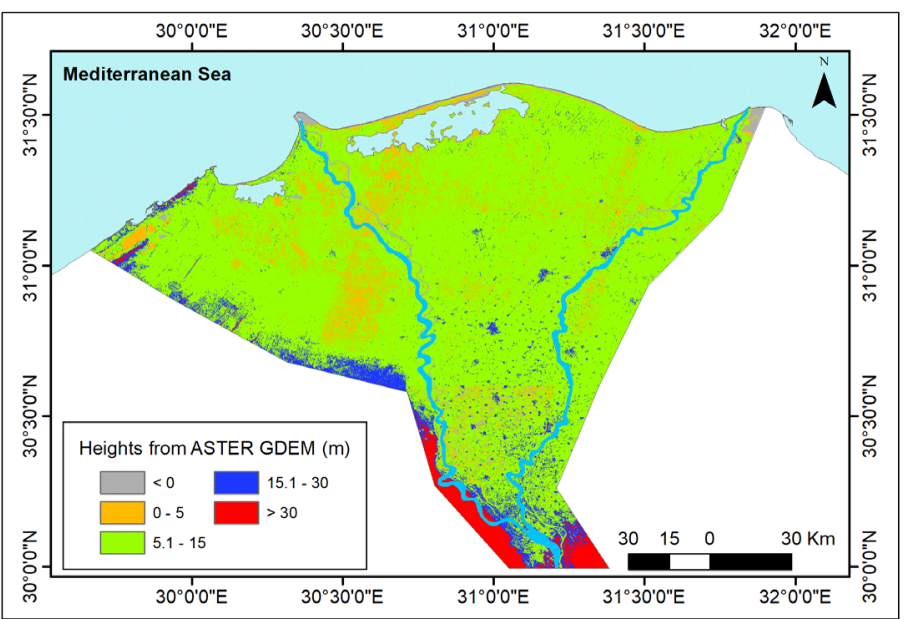

(a)

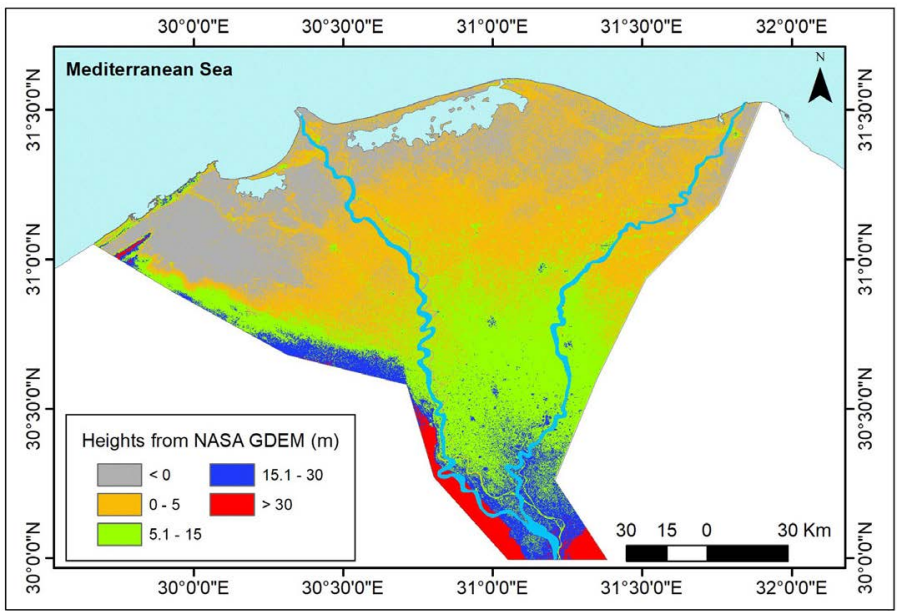

(b) 


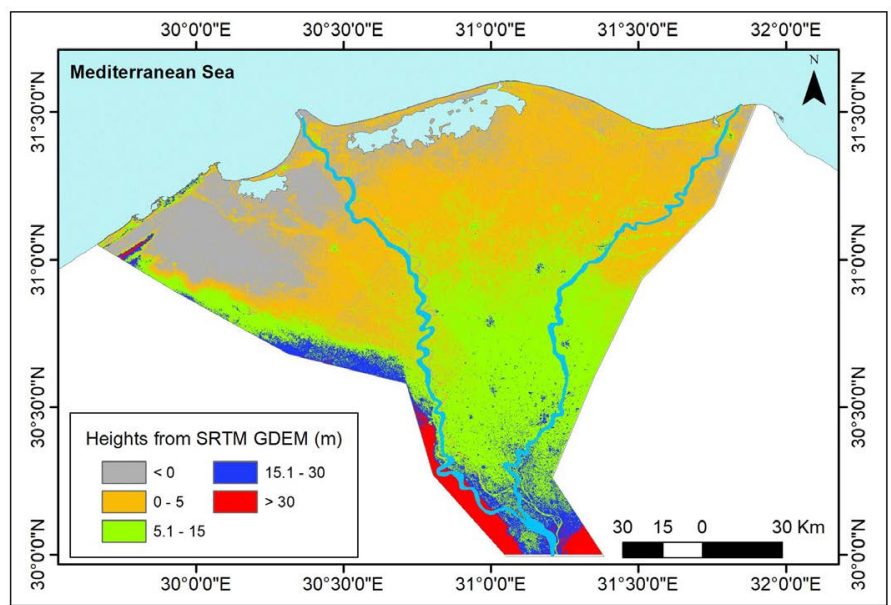

(c)

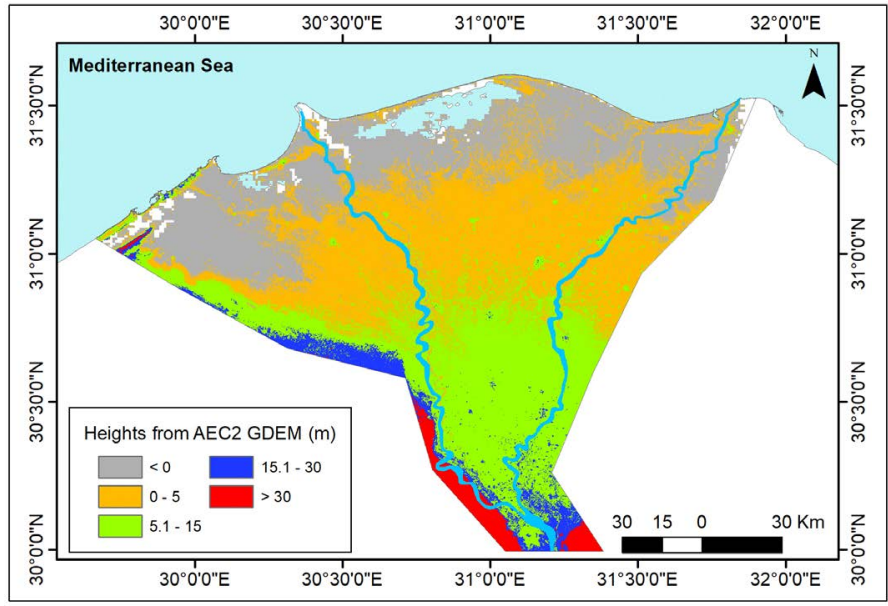

(d)

Figure 3. Topography of Nile delta from GDEMs. (a) ASTER GDEM; (b) NASADEM GDEM; (c) SRTM GDEM; (d) AEC2 GDEM.

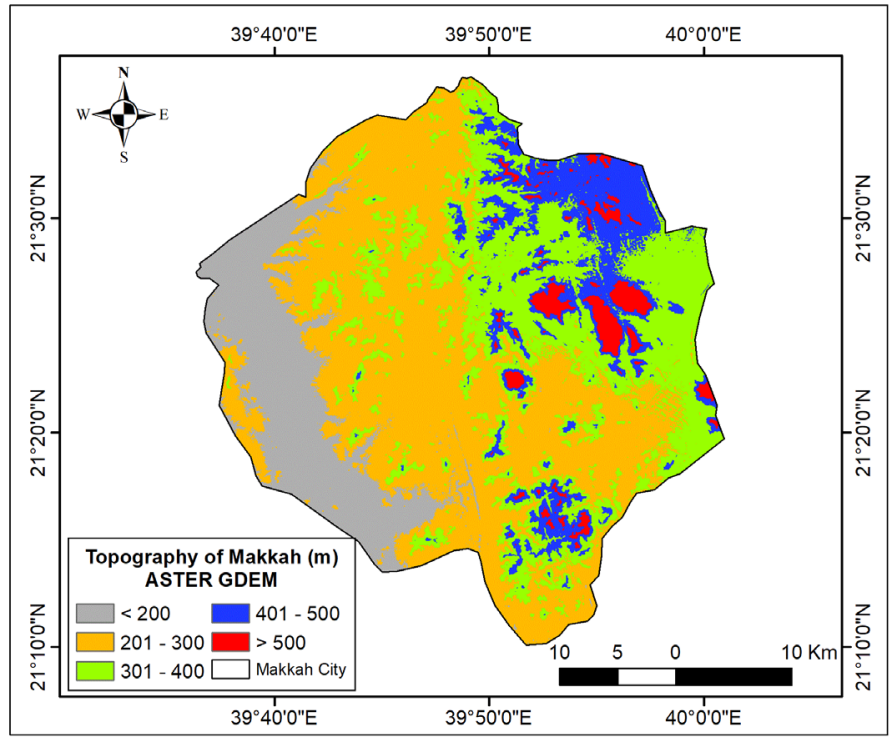

(a) 


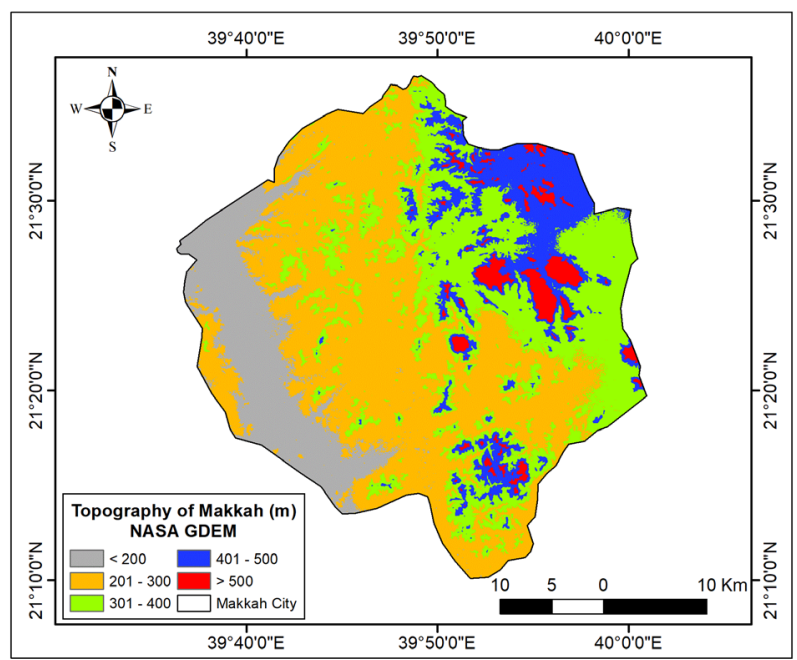

(b)

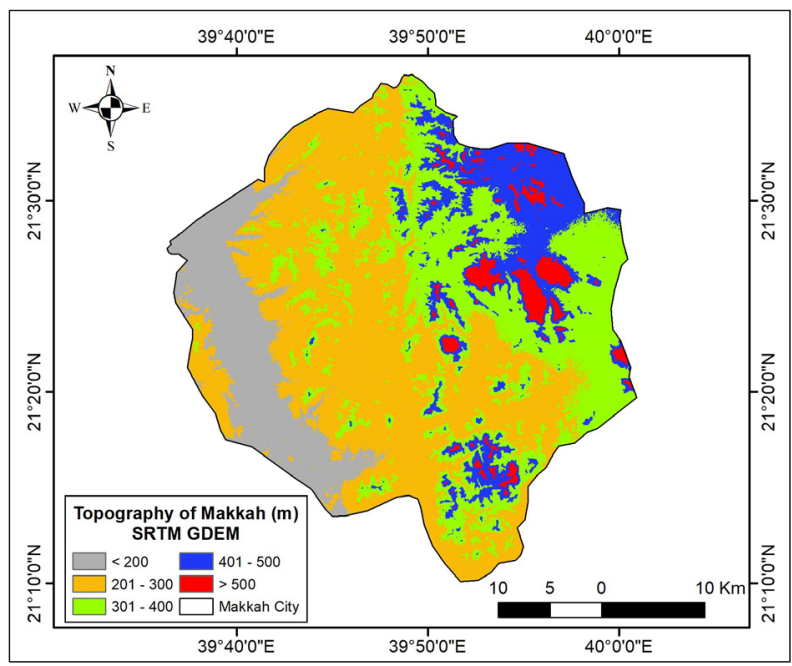

(c)

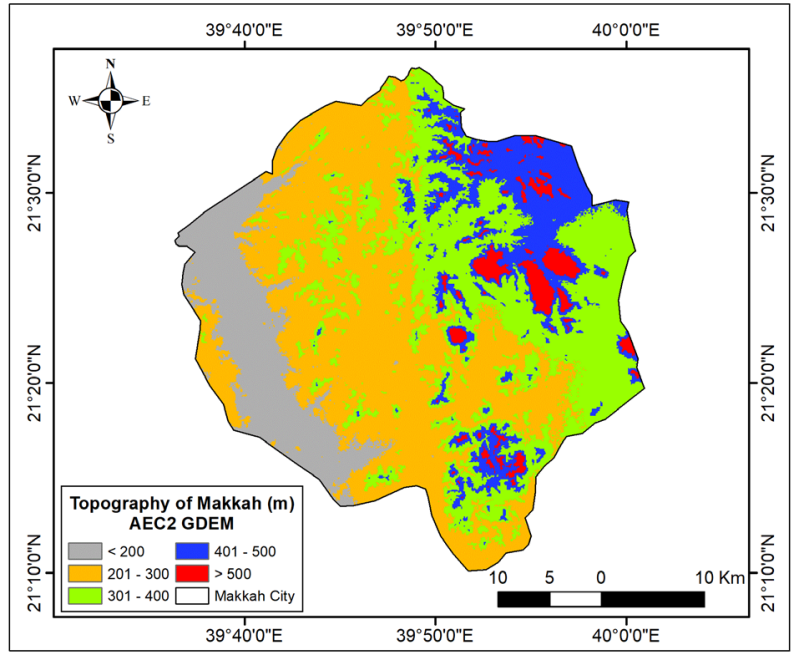

(d)

Figure 4. Topography of Makkah from GDEMs. (a) ASTER GDEM; (b) NASADEM GDEM; (c) SRTM GDEM; (d) AEC2 GDEM. 


\section{Results and Discussion}

Table 4 presents the statistics of observed and GDEM-based heights in the Nile delta region. Generally, it can be noticed that the mean height and the relief (Equation (2)) of only the ASTER GDEM are not close to those of the observed heights, while the other three GDEMs produced relatively comparable estimates. Next, GDEMs' errors have been computed at the known heights of GCPs (Table 5). It can be noticed that the RMS (Equation (1)) of AEC2, STRMGL1, and NASADEM models equal \pm 2.5 meters approximately, while the ASTER model produces the highest RMS of \pm 5.1 meters. Concerning the average heights error, it can be seen that the NASADEM produce the smallest value of 1.8 meters compared to the other three models. The errors distribution has been plotted in Figure 5 for all GDEMs, which emphasizes the overall performance of the investigated GDEMs. It can be realized from that figure that the performance of the ASTER model is far from those of the other models.

Comparing the RMS values of the investigated GDEMs (Table 5) with the required vertical accuracy of utilized datasets (Table 1), it can be concluded (Table 6) that all GDEMs are not suitable for developing contour intervals less than 5 meters. That means that such models should not be utilized for topographic mapping of scale 1:25,000 or larger. For map scale 1:50,000 and 1:100,000 with contour intervals 8 or 10 meters, it can be seen that the ASTER model should be avoided while the other three models meet the required specifications. Additionally, it is clear that for map scales 1:250,000 or smaller, all investigated GDEMs could be utilized.

As mentioned previously, the Nile delta region represents a flat area in terms of topography. Hence, the same steps have been carried out to investigate the performance of GDEMs over Makkah city as a moderate-topography region. Table 7, Table 8 and Figure 6 present the accomplished findings based on the

Table 4. Statistics of height sources in Nile Delta Area.

\begin{tabular}{cccccc}
\hline Item & Minimum & Maximum & Relief & Mean & RMS \\
\hline Observed Heights & -1.925 & 21.950 & 23.875 & 8.532 & \pm 5.814 \\
ASTER v.3 & -12.495 & 32.323 & 44.818 & 10.680 & \pm 5.565 \\
ACE2 & -6.659 & 27.153 & 33.812 & 6.244 & \pm 6.058 \\
STRMGL1 v.3 & -6.900 & 23.561 & 30.461 & 6.561 & \pm 6.139 \\
NASADEM_HGT v.1 & -5.900 & 23.922 & 29.822 & 6.776 & \pm 6.608 \\
\hline
\end{tabular}

Table 5. Statistics of GDEM2 height errors in Nile Delta Area.

\begin{tabular}{cccccc}
\hline Item & Minimum & Maximum & Range & Mean & RMS \\
\hline ASTER v.3 & -19.901 & 18.895 & 38.796 & -2.148 & \pm 5.108 \\
ACE2 & -11.518 & 9.248 & 20.766 & 2.288 & \pm 2.618 \\
STRMGL1 v.3 & -6.320 & 9.500 & 15.820 & 1.971 & \pm 2.584 \\
NASADEM_HGT v.1 & -5.438 & 9.243 & 14.681 & 1.756 & \pm 2.521 \\
\hline
\end{tabular}


Table 6. Validity of GDEM2 for topographic mapping in Nile Delta Area.

\begin{tabular}{|c|c|c|}
\hline Map Scale & Contour Interval (m) & Valid GDEMs \\
\hline $1: 5000$ & 1.25 & \multirow{3}{*}{ NA } \\
\hline $1: 10,00$ & $2.0 / 5.0$ & \\
\hline & 5.0 & \\
\hline $1: 50,000$ & $8.0 / 10.0$ & \multirow[t]{2}{*}{ ACE2, SRTMGL1 v.3, NASADEM_HGT v.1 } \\
\hline & 10.0 & \\
\hline $1: 100,000$ & 20.0 & \multirow[b]{2}{*}{ ASTER v.3, ACE2, SRTMGL1 v.3, NASADEM_HGT v.1 } \\
\hline $1: 250,000$ & $\begin{array}{l}25.0 \\
50.0\end{array}$ & \\
\hline
\end{tabular}

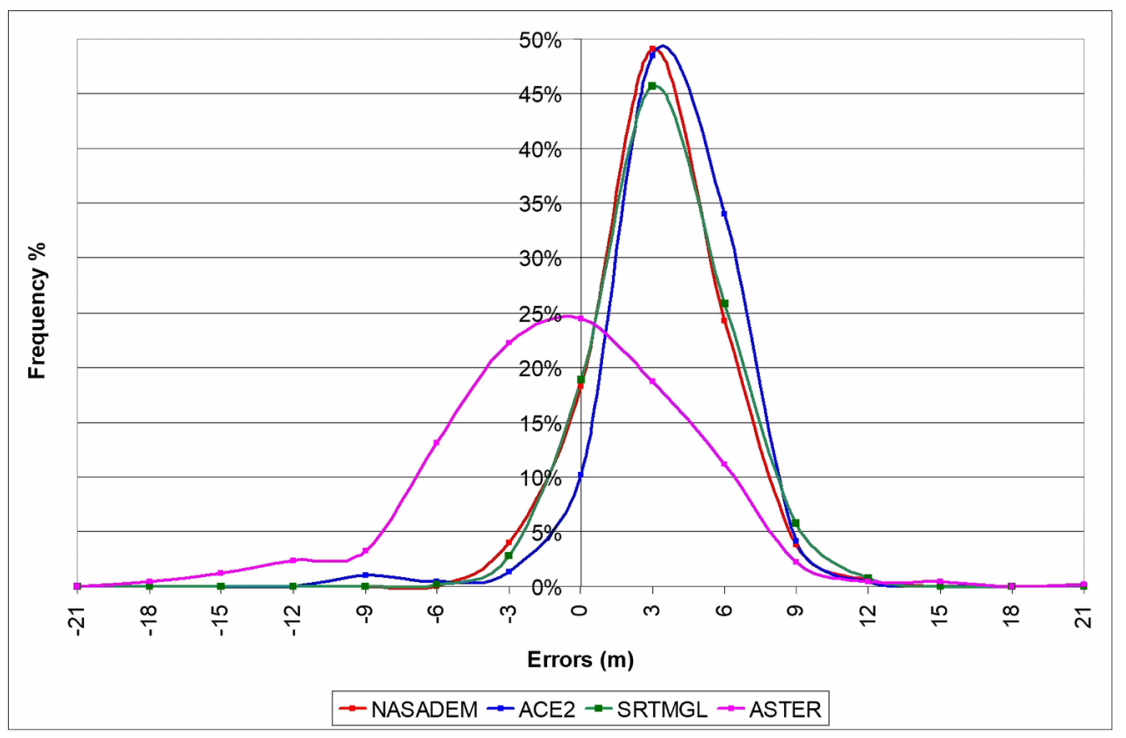

Figure 5. Histograms of height errors of GDEM2 in Nile delta area.

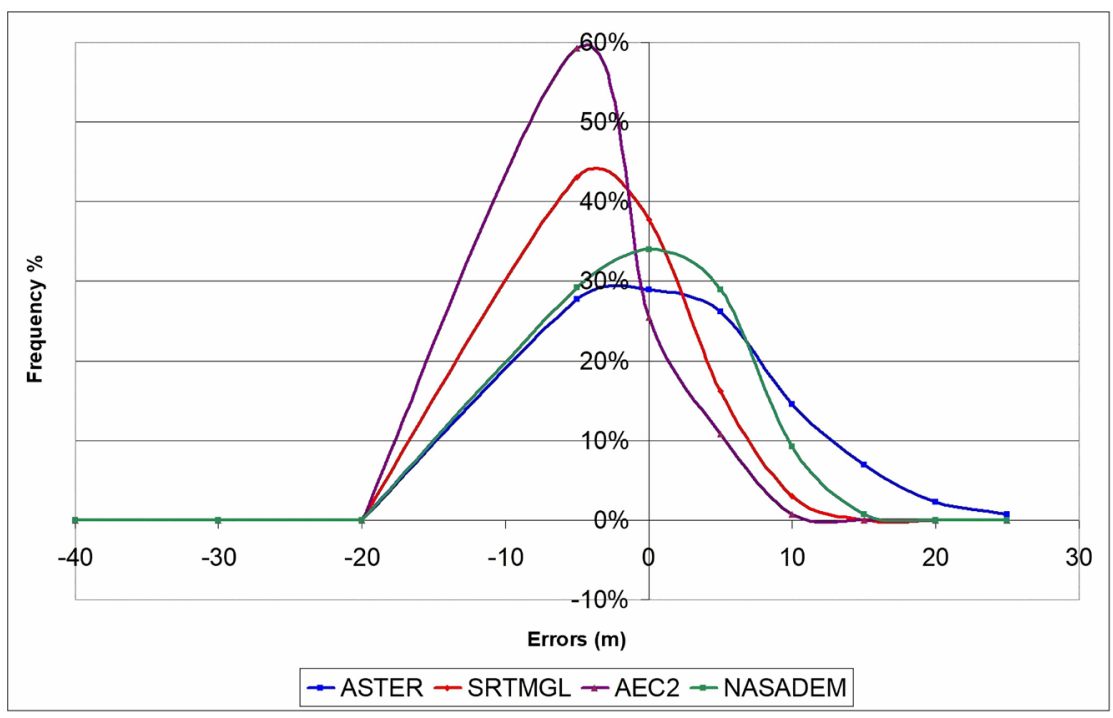

Figure 6. Histograms of height errors of GDEM2 in Makkah area. 
Table 7. Statistics of height sources in Makkah Area.

\begin{tabular}{cccccc}
\hline Item & Minimum & Maximum & Relief & Mean & RMS \\
\hline Observed Heights & 147.135 & 423.187 & 274.823 & 272.146 & \pm 59.189 \\
ASTER v.3 & 144.185 & 419.008 & 274.823 & 272.146 & \pm 59.189 \\
ACE2 & 150.695 & 422.845 & 272.150 & 278.933 & \pm 60.068 \\
STRMGL1 v.3 & 140.482 & 424.860 & 284.378 & 276.082 & \pm 59.541 \\
NASADEM_HGT v.1 & 148.267 & 419.924 & 271.657 & 273.295 & \pm 59.005 \\
\hline
\end{tabular}

Table 8. Statistics of GDEM2 height errors in Makkah Area.

\begin{tabular}{cccccc}
\hline Item & Minimum & Maximum & Range & Mean & RMS \\
\hline ASTER v.3 & -18.456 & 22.830 & 41.286 & -0.334 & \pm 7.996 \\
ACE2 & -34.463 & 5.948 & 40.412 & -7.121 & \pm 6.675 \\
STRMGL1 v.3 & -16.680 & 7.820 & 24.500 & -4.270 & \pm 5.161 \\
NASADEM_HGT v.1 & -13.839 & 11.219 & 25.058 & -1.483 & \pm 5.243 \\
\hline
\end{tabular}

available datasets. It can be noticed from Table 7 that only the SRTMGL1 model produced relief estimates far from the observed precise one. For the mean height error, only the ACE2 model differs a little bit from the other three GDEMs. However, the results of height differences over known GCPs (Table 8) are more important to analyze the GDEMs precision. It can be realized that NASADEM and SRTMGL produced the smallest RMS values, while the ASTER model gives the largest one. Comparing error histograms over both study areas (Figure 5 and Figure 6), it can be noticed that the performance of the investigated GDEMs is more precise over the flat region in the Nile delta than that of the moderate-topography region in Makkah city. This conclusion is evident, too, when comparing the RMS values of GDEMs over both regions. In the Nile delta, the RMS ranges from \pm 2.5 and \pm 5.0 meters, while in Makkah RMS varies between \pm 5.2 and \pm 8.0 meters approximately. Hence, it can be concluded that the accuracy of the investigated GDEMs is almost one order worse in moderate topography regions than flat areas.

Again, a comparison has been performed between the RMS values of the investigated GDEMs in Makkah (Table 8) with the required vertical accuracy of utilized datasets (Table 1). It can be seen from Table 9 that all GDEMs are not suitable for developing contour intervals less than 5 meters. That means that such models are not suitable for topographic mapping of scale 1:50,000 or larger with contour intervals equal or less than 10 meters. For map scale 1:100,000 with contour intervals of 20 meters, it can be seen that the ASTER model should be avoided while the other three models meet the required specifications. From Figure 6, it can be seen that ASTER produced the least-precise frequency pattern of errors over checkpoints compared to other GDEMs. Additionally, it is clear that for map scales 1:250,000 or smaller with contour intervals more than 25 meters, all investigated GDEMs could be utilized. Furthermore, it can be concluded 
Table 9. Validity of GDEM2 for topographic mapping in Makkah Area.

\begin{tabular}{|c|c|c|}
\hline Map Scale & Contour Interval (m) & Valid GDEMs \\
\hline $1: 5000$ & 1.25 & \multirow{5}{*}{ NA } \\
\hline $1: 10,00$ & $2.0 / 5.0$ & \\
\hline 102500 & 5.0 & \\
\hline $1: 50,000$ & $8.0 / 10.0$ & \\
\hline \multirow{2}{*}{$1: 100,000$} & 10.0 & \\
\hline & 20.0 & ACE2, SRTMGL1 v.3, NASADEM_HGT v.1 \\
\hline $1: 250,000$ & $\begin{array}{l}25.0 \\
50.0\end{array}$ & ASTER v.3, ACE2, SRTMGL1 v.3, NASADEM_HGT v.1 \\
\hline
\end{tabular}

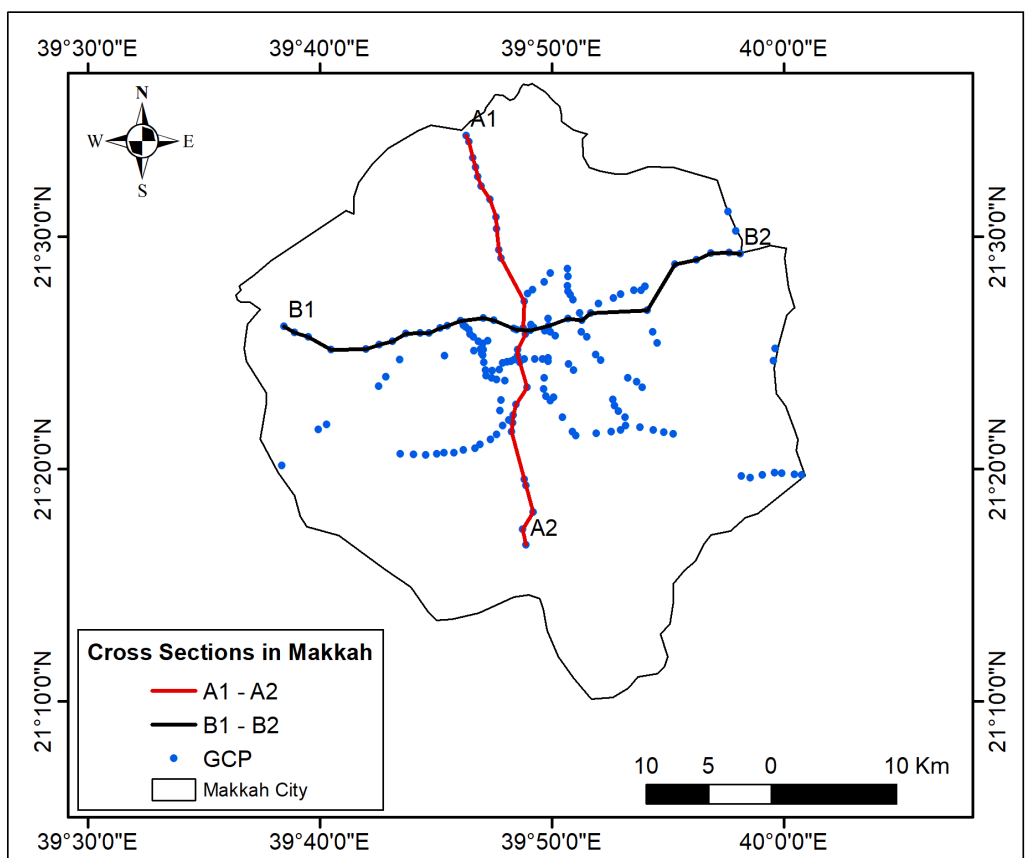

Figure 7. Cross sections in Makkah area.

from Table 6 and Table 9 that the utilization of GDEMs in topographic mapping differs significantly between flat and hilly spatial regions.

For analyzing estimated GDEM-based slopes (Equation (3)) and topographic profiles, two cross-sections have been constructed in Makkah city based on the available GCPs. The first one (B1-B2) runs from west to east passing by $28 \mathrm{GCP}$, while the other cross-section (A1-A2) runs from north to south along 27 GCP (Figure 7). The length of both cross-sections is approximately 35 kilometers. To investigate the validity of GDEM-based slope estimation along a cross-section, each one is divided by 5-kiolmeters intervals. Equation (4) has been applied to compute the slope validity factor (SV) at each interval for both sections. Figure 8 depicts the accomplished results, that reveals two important remarks. First, all GDEM-based slopes do not match with the actual slopes from known GCP over 


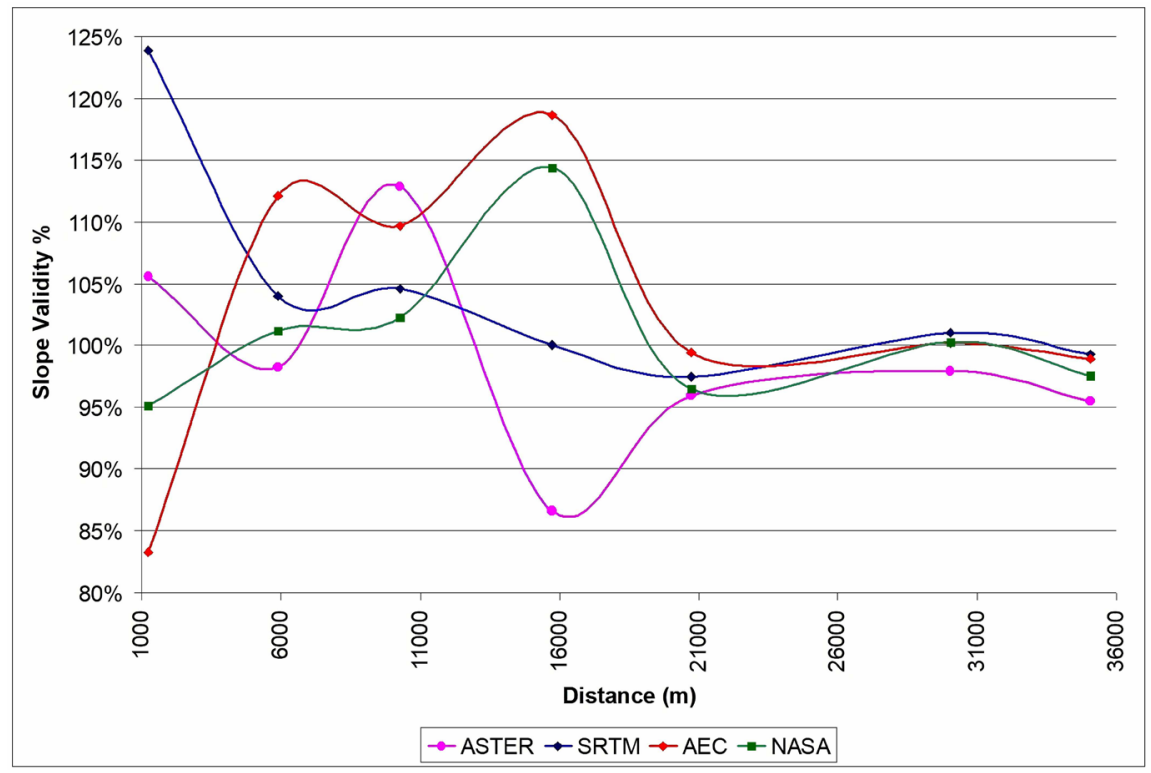

(a)

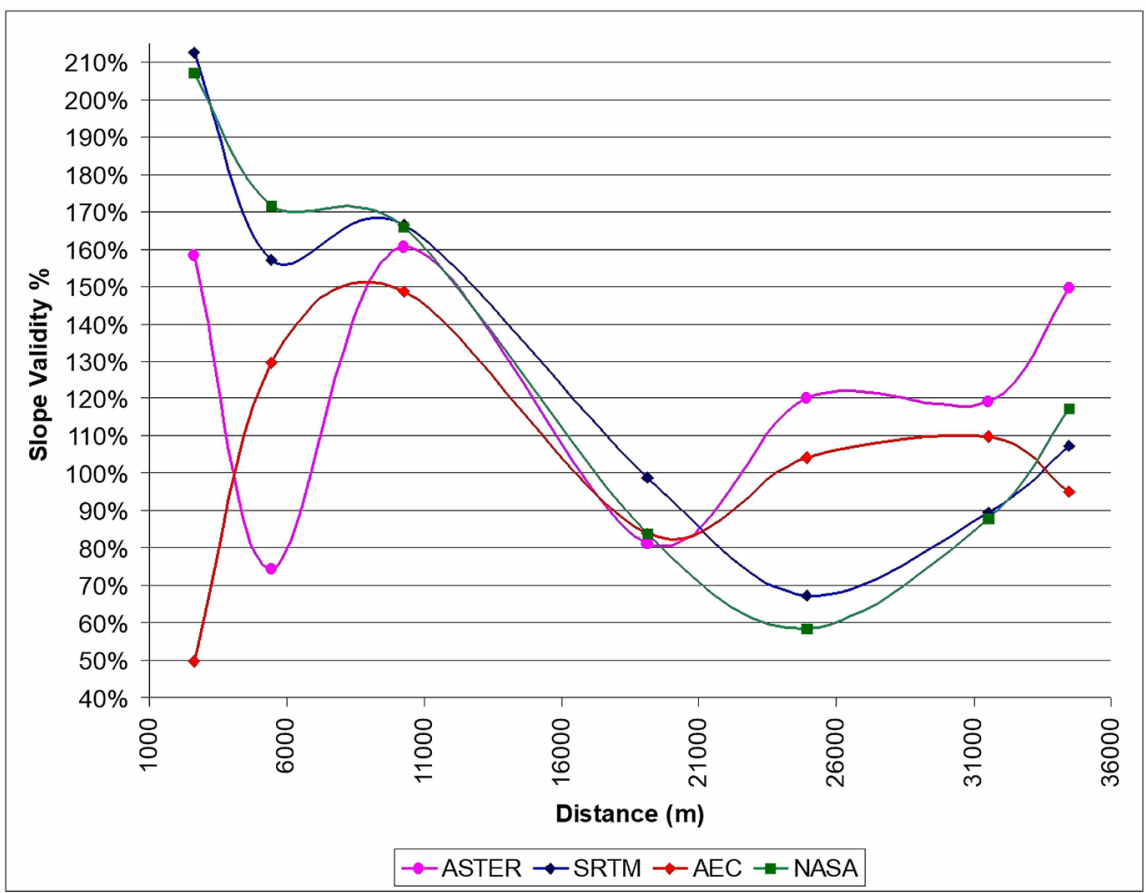

(b)

Figure 8. Slope validity over cross sections in Makkah area.

cross section's length up to 25 and 30 kilometers for both profiles respectively. Second, GDEM-based slopes for profiles more than 30-kilometers length close to the accurate slopes within a value of $\pm 10 \%$ approximately. Hence, it can be concluded that GDEMs should not be utilized for developing topographic cross-sections less than 30 kilometers. Additionally, it can be said that GDEMs-based topographic profiles should not be utilized in engineering and geomatics applications that need precise heights and slopes information. Consequently, the attained 
findings of the current study, based on the available datasets, showed that GDEMs are not the appropriate heights' source for topographic mapping at medium and large map scales, and could not be utilized for accurate topographic profiling in precise engineering and geomatics applications.

\section{Conclusions}

Open-source GDEMs have been utilized in the last couple of decades for topographic mapping and several geomatics activities. Specifications of topographic maps rely on the appropriate contour interval on each map scale and the accuracy of the utilized heights information. Examples of topographic mapping standards from several countries have been collected and investigated. The current study aims to examine the validity and reliability of GDEMs-based heights for topographic mapping at several map scales. Particularly, it investigates the performance of GDEMS for contour intervals, slope estimation, and generating topographic profiles needed for engineering and environmental applications in both Egypt and Saudi Arabia.

Two study areas have been utilized to investigate the performance of four GDEMs for topographic mapping at flat and moderate-topography patters. Based on the available datasets in the two study areas, several important findings have been attained. Using known checkpoints, it has been found that the accuracy of investigated GDEMs ranges from \pm 2.5 and \pm 5.1 meters in the Nile delta region, while it varies between \pm 5.1 and \pm 8.0 meters in the Makkah area. That indicates that the utilization of GDEMs in topographic mapping differs significantly between flat and hilly spatial regions. Based on the contour interval required on several topographic map scales, it has been found that GDEMs are not appropriate for generating contour interval less than 10 meters. Consequently, it is suggested not to use GDEMs for developing topographic maps of scale 1:25,000 or larger in flat regions and map scale 1:50,000 or larger in hilly regions.

In terms of slope estimation and developing a topographic profile, the obtained results revealed that all GDEM-based slopes do not fit the actual slopes from known GCP over cross-section lengths up to 30 kilometers. It has been also observed that GDEM-based slopes for profiles more than 30-kilometers length close to the accurate slopes within a value of $\pm 10 \%$ approximately. As a result, it could be concluded that GDEMs are not the appropriate heights' source for topographic mapping at medium and large map scales, and could not be utilized for topographic profiling in precise engineering and geomatics applications.

\section{Conflicts of Interest}

The authors declare no conflict of interest regarding the publication of this paper.

\section{References}

[1] Ogania, J., Puno, G., Alivio, M. and Taylaran, J. (2019) Effect of Digital Elevation Model's Resolution in Producing Flood Hazard Maps. Global Journal of Environ- 
mental Science and Management, 5, 95-106.

[2] Seleem, T. (2013) Analysis and Tectonic Implication of DEM-Derived Structural Lineaments, Sinai Peninsula, Egypt. International Journal of Geosciences, 4, 183-201. https://doi.org/10.4236/ijg.2013.41016

[3] Boulton, S.J. and Stokes, M. (2018) Which DEM Is Best for Analyzing Fluvial Landscape Development in Mountainous Terrains? Geomorphology, 310, 168-187. https://doi.org/10.1016/j.geomorph.2018.03.002

[4] Hasan, E., Khan, S. and Hong, Y. (2015) Investigation of Potential Sea Level Rise Impact on the Nile Delta, Egypt Using Digital Elevation Models. Environmental Monitoring and Assessment, 187, 649. https://doi.org/10.1007/s10661-015-4868-9

[5] Ouerghi, S., ELsheikh, R., Achour, H. and Bouazi, S. (2015) Evaluation and Validation of Recent Freely-Available ASTER-GDEM V.2, SRTM V.4.1 and the DEM Derived from Topographical Map over SW Grombalia (Test Area) in North East of Tunisia. Journal of Geographic Information System, 7, 266-279. https://doi.org/10.4236/jgis.2015.73021

[6] Al-Karagy, E. and Dawod, G. (2021) Optimum Combinations of GGM and DEM Models for Precise National Geoid Development. Proceedings of Engineering and Technology Innovation.

[7] Dawod, G. and Al-Ghamdi, K. (2017) Reliability of Recent Global Digital Elevation Models for Geomatics Applications in Egypt and Saudi Arabia. Journal of Geographic Information System, 9, 685-698. https://doi.org/10.4236/jgis.2017.96043

[8] Kulp, S. and Strauss (2016) Global DEM Errors Underpredict Coastal Vulnerability to Sea Level Rise and Flooding. Frontiers in Earth Science, 4, 36. https://doi.org/10.3389/feart.2016.00036

[9] Patel, A., Katiyar, S. and Prasad, V. (2016) Performances Evaluation of Different Open Source DEM Using Differential Global Positioning System (DGPS). The Egyptian Journal of Remote Sensing and Space Sciences, 19, 7-16. https://doi.org/10.1016/j.ejrs.2015.12.004

[10] Hu, Z., Peng, J., Hou, Y. and Shan, J. (2017) Evaluation of Recently Released Open Global Digital Elevation Models of Hubei, China. Remote Sensing, 9, 262.

https://doi.org/10.3390/rs9030262

[11] Church, J., Clark, P., Cazenave, A., Gregory, J, Jevrejeva, S., Levermann, A., Merrifield, M., Milne, G., Nerem, R., Nunn, P., Payne, A., Pfeffer, W., Stammer, D. and Unnikrishnan, A. (2013) Sea Level Change. In: Climate Change 2013: The Physical Science Basis. Contribution of Working Group I to the Fifth Assessment Report of the Intergovernmental Panel on Climate Change (IPCC), Cambridge University Press, Cambridge, 1137-1216.

[12] Abdel-Aziz, T., Dawod, G. and Ebaid, H. (2020) DEMs and Reliable Sea Level Rise Risk Monitoring in Nile Delta, Egypt. Discover Sustainability, 1, Article No. 6. https://doi.org/10.1007/s43621-020-00006-7

[13] Sande, B., Lansen, J. and Hoyng, C. (2012) Sensitivity of Coastal Flood Risk Assessments to Digital Elevation Models. Water, 4, 568-579.

https://doi.org/10.3390/w4030568

[14] Zhang, J. and Chu, X. (2015) Impact of DEM Resolution on Puddle Characterization: Comparison of Different Surfaces and Methods. Water, 7, 2293-2313. https://doi.org/10.3390/w7052293

[15] Survey of India (2009) Handbook of Topography. http://www.iism.nic.in/Documents/soichapter-xi.pdf 
[16] ASPRS (American Society for Photogrammetry and Remote Sensing) (2015) ASPRS Positional Accuracy Standards for Digital Geospatial Data. Edition 1, Version 1. Photogrammetric Engineering and Remote Sensing, 81, A1-A26. https://doi.org/10.14358/PERS.81.3.A1-A26

[17] Geoscience Australia (2021) National Topographic Database Structure and Specifications.

http://www.ga.gov.au/mapspecs/topographic/v6/section1.html\#MinimumPlanimetr icElevationAccuracies

[18] JICA (Japan International Cooperation Agency) (2013) The Study on Establishing Digital Topographic Database for Bujumbura City, Burundi. A Technical Report. https://openjicareport.jica.go.jp/pdf/12121588_01.pdf

[19] NDMA (National Disaster Management Authority) (2016) National Technical Document Fir Establishing Cartographic Database in India: Generation of Large Scale (1:10,000 and 1:2,000 and Leaser) Maps for Disaster Management and Planning. A Technical Report.

http://www.ndma.gov.in/sites/default/files/PDF/Technical\%20Documents/nationaltechnical-document-for-establishing-cartographic-base-in-india.pdf

[20] USGS (US Geological Survey) (1999) Map Accuracy Standards: Fact Sheet FS-171-99. A Technical Report. https://pubs.usgs.gov/fs/1999/0171/report.pdf

[21] Qatar GIS Center (2009) Qatar Survey Manual Version 1. A Technical Report, Qatar.

[22] ESA (Egyptian Survey Authority) (2020) Technical Specifications of Surveying and Mapping: Part 2: Topographic Maps. A Technical Report, Giza.

[23] Khal, M., Algouti, A., Algouti, A., Akdim, N., Stankevich, S. and Menenti, M. (2020) Evaluation of Open Digital Elevation Models: Estimation of Topographic Indices Relevant to Erosion Risk in the Wadi M'Goun Watershed, Morocco. Geosciences, 6, 231-257. https://doi.org/10.3934/geosci.2020014

[24] Cahyaningsih, C., Crensonni, P., Suryadi, A., Kausarian, H., Choanji, T., Yuskar, Y. and Putra, D. (2019) Geomorphology and Structural Geology Characterization of Landslide Prone Area in Riau-West of Sumatra Highway. IOP Conference Series Materials Science and Engineering, 536, Article ID: 012063. https://doi.org/10.1088/1757-899X/536/1/012063

[25] Mokarram, M. and Hojati, M. (2016) Comparison of Landform Classifications of Elevation, Slope, Relief and Curvature with Topographic Position Index in the South of Bojnoord. Ecopersia, 2, 1343-1357.

https://doi.org/10.18869/modares.ecopersia.4.2.1343

[26] USGS (US Geological Survey) (2021a) SRTMGL1 V003. https://lpdaac.usgs.gov/products/srtmgl1v003

[27] Smith, R. and Berry, P. (2019) Altimeter Corrected Elevations, Version 2. https://sedac.ciesin.columbia.edu/data/set/dedc-ace-v2

[28] US Open Data Catalouge (2021) ASTER Digital Elevation Model V003. https://catalog.data.gov/dataset/aster-digital-elevation-model-v003

[29] USGS (US Geological Survey) (2021b) NASADEM_HGT: Merged DEM Global 1 Arc Second v. 1. https://lpdaac.usgs.gov/products/nasadem_hgtv001 